\title{
Myocardial Remodeling in Congenital Heart Disease
}

\author{
Vera Demarchi Aiello and Maria Angélica Binotto \\ Instituto do Coração do Hospital das Clínicas - FMUSP - São Paulo, SP - Brazil
}

Myocardial structure in congenital heart defects had not been extensively analyzed until recently. It was not until 15 or 20 years ago that the first studies appeared in the literature.

Back in the first half of the last century, the first challenge in the study of congenital heart defects was the understanding of the morphology and the approach to diagnosis. Morphology has now been studied for more than one century, and nowadays virtually all the anomalies have their anatomy well clarified.

With the advent of cardiac surgery in the 1950s, the next step was to correct or palliate the defects, and this, to a great extent, has also been achieved today.

In parallel to the improvement of diagnostic methods and surgical technique, clinical management also experienced an enormous development during the last five decades. Patients with heart defects, now surviving to adolescence and adulthood, required the specialization of clinicians and surgeons, in order to understand well the late outcome of their diseases and to treat their residual lesions.

The next goals to be achieved are the prevention of myocardial remodeling with an early approach to the defects and the understanding of the previous or intrinsic myocardial abnormalities.

The distorted cardiac morphology in congenital heart defects leads to variable hemodynamic consequences that promote myocardial adaptation and may, eventually, induce heart failure ${ }^{1}$. Since these particular conditions of volume and pressure overload are present from cardiac morphogenesis, the remodeling process occurs in parallel to the growth process of the heart, before and after birth.

Although the term "myocardial remodeling" has been sometimes taken as a synonym to "cardiac dilation", its concept implies a series of changes in size, shape and function of the heart, that histologically correspond to myocyte hypertrophy or loss (due to necrosis or apoptosis), changes in the amount of extracellular matrix (mainly fibrosis), and changes in the microvasculature, such as capillary proliferation ${ }^{2}$. Particularly in small children, the mechanism of myocyte hyperplasia may also take part in the process.

Histological myocardial remodeling has been studied in different types of congenital defects, although not extensively.

\section{Key words}

Heart defects, congenital; myocardium.

Mailing address: Vera Demarchi Aiello •

Av. Dr. Enéas Carvalho Aguiar, 44 - São Paulo, SP - Brazil

E-mail: vera.aiello@incor.usp.br

Manuscript received December 5, 2006; revised received January 3, 2007

accepted January 3, 2007.
An increase in interstitial myocardial fibrosis was documented in tricuspid atresia ${ }^{3,4}$, tetralogy of Fallot ${ }^{5}$, Ebstein's anomaly ${ }^{6}$ and in pulmonary atresia with intact ventricular septum ${ }^{7}$, while in hypoplastic left heart syndrome the described change of extracellular matrix is toward a decreased percentage of collagen per field when compared to normal ${ }^{8}$. Fibrosis deposition is thought to result in increased myocardial stiffness, which can impair ventricular filling. A transmural variation in the quantity of fibrosis was described, the subendocardium being the region with the highest percentage of fibrosis ${ }^{3}$. The pathogenesis of such increased matrix deposition is not fully explained, but the presence of chronic hypoxia and an inadequacy of coronary/capillary supply, especially in the subendocardial region, are believed to play an important role. Another important finding in patients with tricuspid atresia was a positive correlation between the amount of myocardial interstitial fibrous tissue and $\mathrm{age}^{4}$.

Myocyte hypertrophy is a non-specific response to volume or pressure overload of the ventricles. Characteristically, pressure overload leads to a situation where the wall thickness increases but the chamber radius remains constant or even diminishes (concentric hypertrophy), as a result of growth in transverse myocyte diameter. On the other hand, volumeoverload hypertrophy progresses with a proportional growth in the transverse and longitudinal dimensions of the myocytes and chamber dilation (eccentric hypertrophy). Wall thickness is not necessarily increased, and may be normal or thinned. Myocyte disarray can also accompany a proportion of cases showing hypertrophy, as a non-specific feature ${ }^{7}$. Induction of cardiac hypertrophy by means of pressure-overload may be useful in cases of transposition of the great arteries, which is achieved by pulmonary artery banding before surgical correction (Jatene operation, also known as two-stage arterial switch operation-ASO $)^{9,10}$. There is some debate about potential adverse effects of such induced hypertrophy, since late results have demonstrated that myocardial contractility is lower after the two-stage ASO than after a primary repair ${ }^{11}$.

Another point of discussion in cardiac remodeling is the possibility of cardiomyocytes re-entering the cell cycle. Several studies have demonstrated that cardiac myocytes do synthesize DNA under certain conditions ${ }^{12}$, but whether the number of cells or just the number of nuclei increases has not been completely answered ${ }^{13}$. Increases in myocyte cell number appear to occur in the early postnatal period, as demonstrated experimentally ${ }^{14}$. Thus, it is possible that human hearts with congenital defects can also adapt to different hemodynamic conditions after birth by means of cell hyperplasia.

Besides proportionally accompanying physiological cardiac growth, capillaries are involved in all the mechanisms of myocardial adaptation under adverse conditions ${ }^{15}$. In some circumstances leading to cardiac hypertrophy, the inadequacy 
in their number is believed to increase the potential for ischemic damage. Although hypoxia is considered to be an effective stimulus for endothelial cell growth, experimental data of capillary supply adaptation in response to hypoxia are controversial. Numbers of capillaries were considered inadequate in hearts of humans presenting hypoplastic left heart syndrome ${ }^{16}$ and in tricuspid atresia ${ }^{4}$. This may be interpreted as an inherent abnormality with implications for ventricular development or indicate a greater vulnerability of these hearts to ischemia.

In summary, when thinking about myocardial remodeling in congenital heart disease, we should consider that: it is an ongoing process occurring since intrauterine life, because the defects are present since morphogenesis; it develops in parallel with the normal growth process, which makes it unique and different from remodeling in mature hearts, and it continues even after surgery, because children continue to grow and may have residual defects.

\section{References}

1. Bolger AP, Gatzoulis MA. Towards defining heart failure in adults with congenital heart disease. Int J Cardiol. 2004; 97: 15-23.

2. Cohn JN, Ferrari R, Sharpe N. Cardiac remodeling-concepts and clinical implications: a consensus paper from am international forum on cardiac remodeling. J Am Coll Cardiol. 2000;35:569-82.

3. Ho SY, Jackson M, Kilpatrick L, Smith A, Gerlis LM. Fibrous matrix of ventricular myocardium in tricuspid atresia compared with normal heart: a quantitative analysis. Circulation. 1996;94:1642-6.

4. Binotto MA, Higuchi ML, AielloVD. Left ventricular remodeling in hearts with tricuspid atresia: morphologic observations and possible basis for ventricular dysfunction after surgery. JThorac Cardiovasc Surg. 2003; 126: 1026-32.

5. Peters TH, Sharma HS, Yilmaz E, Bogers AJ. Quantitative analysis of collagens and fibronectin expression in human right ventricular hypertrophy. Ann NY Acad Sci. 1999; 874: 278-85.

6. Akiba T, Becker AE. Disease of the left ventricle in pulmonary atresia with intact ventricular septum: the limiting factor for long-lasting successful surgical intervention? J Thorac Cardiovasc Surg. 1994; 108: 1-8.

7. Celermajer DS, Dodd SM, Greenwald SE, Wyse RK, Deanfield JE. Morbid anatomy in neonates with Ebstein's anomaly of the tricuspid valve: pathophysiologic and clinical implications. J Am Coll Cardiol. 1992; 19 1049-53.

8. Salih C, McCarthy KP, Ho SY. The fibrous matrix of ventricular myocardium in hypoplastic left heart syndrome: a quantitative and qualitative analysis. Ann Thorac Surg. 2004; 77: 36-40.
9. Salih C, Sheppard MN, Ho SY. Morphometry of coronary capillaries in hypoplastic left heart syndrome. Ann Thorac Surg. 2004;77:903-7.

10. Wong K, Boheler KR, Petrou M, Yacoub MH. Pharmacological modulation of pressure-overload cardiac hypertrophy: changes in ventricular function, extracellular matrix, and gene expression. Circulation. 1997, 96 (7): $2239-46$.

11. Dias CA, Assad RS, Caneo LF, Abduch MC, Aiello VD, Dias AR, et al. Reversible pulmonary trunk banding. II. An experimental model for rapid pulmonary ventricular hypertrophy. JThorac Cardiovasc Surg. 2002; 124: 999-1006.

12. Boutin C, Wernovsky G, Sanders SP, Jonas RA, Castaneda AR, Colan SD. Rapid two-stage arterial switch operation: evaluation of left ventricular systolic mechanics late after an acute pressure overload stimulus in infancy. Circulation. 1994; 90: 1294-303.

13. Beltrami CA, Di Loreto C, Finato N, Rocco M, Artico D, Cigola E, et al. Proliferating cell nuclear antigen (PCNA), DNA synthesis and mitosis in myocytes following cardiac transplantation in man. J Mol Cell Cardiol. 1997:29:2789-802.

14. Anversa P, Kajstura J. Ventricular myocytes are not terminally differentiated in the adult mammalian heart. Circ Res.1998; 83: 1-14.

15. Reiss K, Cheng W, Ferber A, Kajstura J, Li P, Li B, et al. Overexpression of insulin-like growth factor- 1 in the heart is coupled with myocyte proliferation in transgenic mice. Proc Natl Acad Sci USA. 1996; 93: 8630-5.

16. Anversa P, Ricci R, Olivetti G. Coronary capillaries during normal and pathological growth. Can J Cardiol. 1986; 2: 104-13. 